\title{
Inundaciones por zonas funcionales en la subcuenca Santa Rosa Jáuregui, Querétaro, México
}

\author{
Flooding within functional zones in the Santa Rosa Jauregui \\ subwatershed Queretaro, Mexico
}

\author{
Sonia Oreano-Hernández ${ }^{1}$ \\ Universidad Autónoma de Querétaro, México \\ Juan Hernández-Guerrero ${ }^{2}$ \\ Universidad Autónoma de Querétaro, México
}

\begin{abstract}
Resumen
En la periferia urbana de la ciudad de Querétaro, las inundaciones son más frecuentes y peligrosas. El proceso parece acentuarse conforme se altera la estructura y función de las cuencas hidrográficas. El presente trabajo analizó la formación de inundaciones por zonas funcionales en la subcuenca Santa Rosa Jáuregui, Querétaro. Al respecto, se elaboraron zonas funcionales con variables hidrogeológicas en un sistema de información geográfica, se definieron unidades y secciones con análisis hidrogeomorfológico y se recopilaron registros de inundaciones con trabajo hemerográfico. Los resultados señalan que la zona funcional alta tiene una estructura óptima que beneficia cuenca abajo, pero las alteraciones hidrogeomorfológicas en la zona funcional media favorecen la formación de inundaciones en la zona funcional baja, la cual también cuenta con la urbanización de la llanura de inundaciones. A través de zonas funcionales, se determinó que las inundaciones resultan de procesos sistémicos y acumulativos que pueden ser utilizados en la gestión y mitigación de riesgo.
\end{abstract}

Palabras clave: Zona funcional, inundación, subcuenca, periferia urbana.

1 Maestra en Gestión Integrada de Cuencas. Universidad Autónoma de Querétaro. Facultad de Ciencias Naturales. Campus Aeropuerto, México. Carretera a Chichimequillas s/n, Ejido Bolaños. C.P. 76140. Correo electrónico: sandyoreano@gmail.com iD https://orcid.org/0000-0002-7205-9234

2 Doctor en Geografía. Universidad Autónoma de Querétaro. Facultad de Ciencias Naturales. Campus Aeropuerto, México. Carretera a Chichimequillas s/n, Ejido Bolaños. C.P. 76140. Correo electrónico: juan. hernandez@uaq.mx iD https://orcid.org/0000-0002-4220-7216 


\begin{abstract}
In the urban periphery of the city of Queretaro, Mexico, floods are becoming increasingly frequent and dangerous. The process seems to be accentuated as the structure and function of hydrographic watersheds are altered. The present work analyzed the occurrence of floods within functional zones in the Santa Rosa Jauregui subwatershed in the state of Queretaro, Mexico. In this regard, functional zones were elaborated with hydrogeological variables in a Geographic Information System, units and sections were defined by employing hydrogeomorphological analysis, and flood records were compiled from hemerographic research. The results indicate that the high functional zone possesses an optimal structure that benefits the watershed downstream, however, hydrogeomorphological alterations in the middle functional zone favor the occurrence of floods in the low functional zone, which finds its floodplain in an urbanized state. Through consideration of functional zones, it was determined that floods result from systemic and cumulative processes whose awareness and consideration can be used for risk management and mitigation purposes.
\end{abstract}

Keywords: Functional zone; flood; subwatershed; urban periphery.

\title{
Introducción
}

En los temas de construcción y gestión del riesgo asociado con inundaciones, la relación entre amenazas de origen hidrometeorológico y las actividades humanas son determinantes, pero también la propia estructura y función hídrica del lugar, pues al combinarse con alteraciones, exposición y vulnerabilidad, se fomenta la recurrencia y peligrosidad de los eventos y, por ende, la susceptibilidad del lugar (Cutter, Boruff \& Lynn 2003; Hernández, 2013; Zúñiga \& Magaña, 2017).

Los lugares susceptibles a inundaciones deben analizarse como consecuencia de efectos acumulativos y multicausales más allá de la zona de anegación, a los cuales se les brinda poca atención porque parecieran estar ocultos o remotos a donde ocurre el evento (Hernández, Vieyra \& Mendoza, 2012). Por ello, comprender la formación y consecuencias de las inundaciones requiere de estudios no lineales e interdependientes de las condiciones (biofísicas, sociales y culturales), escala y características territoriales (Mavhura, 2019).

Entre la diversidad de unidades territoriales, las cuencas hidrográficas destacan en el análisis de inundaciones por sus funciones dinamizadas por el agua, estructura constituida por subsistemas de elementos complejos y heterogéneos (geológicos, geomorfológicos y edáficos) que permiten analizar la formación de inundaciones de manera sistémica (Reid, 1993; Braud, Fletcher \& Andrieu, 2013; Jáuregui, Aversa \& Salas, 2016). En ese sentido, la comprensión sistémica puede asociarse con zonas funcionales: a) zona alta, definida por la captación de agua de lluvia; b) zona media de 
almacenamiento y transporte de agua; y c) zona baja de descarga y captación de agua (Valdés \& Hernández, 2018, p. 190).

Las zonas funcionales media y baja, según las características del lugar, suelen ser las más recurrentes a la formación de inundaciones, ya que denotan el efecto acumulativo natural de la dinámica pluvial y fluvial de la cuenca a través de escorrentías y descargas sobre ríos y cuerpos de agua perennes y temporales, o bien, sitios de baja pendiente, por esto, cualquier perturbación altera la dinámica (Garrido, Enríquez, Pérez, Luna \& Sánchez, 2009; Valdés \& Hernández, 2018).

En cuencas limítrofes a las ciudades, específicamente en las periferias urbanas, las zonas funcionales media y baja suelen priorizarse a la inversión y ocupación, ya sea por su cercanía con el área urbana central o por su atractivo ambiental (producción de alimentos, regulación de microclimas, abastecimiento hídrico, atracción de biodiversidad y provisión de servicios ambientales), todo ello es interdependiente de la dinámica particular de actividades y funciones naturales, rurales, urbanas e industriales (Braud et al., 2013; Flotemersch et al., 2016; Hernández \& Osorno, 2018). No obstante, esos espacios suelen presentar importantes accidentes del terreno, insuficiente vigilancia, cambios de suelo con poca planeación, tenencia del suelo diferenciada y mínima atención gubernamental (Craig \& Jeffery, 2014; Gunnell, Mulligan, Francis \& Hole 2019). Lo anterior propicia que se modifique el proceso natural y formador de la inundación, al tiempo que la evaluación y actuación se complique debido a los acelerados cambios y actividades (Cardoso \& Fritchy, 2012; Braud et al., 2013; Gunnell et al., 2019).

Al respecto, existen trabajos sobre inundaciones en cuencas hidrográficas de periferias urbanas que estudiaron los efectos ambientales y territoriales (Craig \& Jeffery, 2014; Barbedo, Miguez, Van der Horst \& Marins, 2014; Arroyo, 2018), modelaron inundaciones (Mobayed, 2009; Miller et al., 2014; Califano, Mobilia \& Longobardi, 2015; Oviedo, Morán, Nájera \& Bolívar, 2018) y analizaron la formación e impactos socioeconómicos y culturales (Eakin, Lemer \& Murtinho, 2010; Hernández et al., 2012; Becerra et al., 2013; García \& Hernández, 2015; Michel \& Hernández, 2019). Sin embargo, en los trabajos mencionados, aun cuando se plantea de forma directa e indirecta la dinámica de la cuenca, no se realiza un análisis sistémico para comprender la formación de inundaciones, 
ante lo cual, su análisis por zonas funcionales serían una alternativa en el proceso de mitigación.

Los argumentos mencionados hasta el momento se ejemplifican en la mayoría de las periferias urbanas de ciudades grandes en México, sea por su crecimiento poblacional y físico, competitividad, reconfiguración territorial y ocupación de suelos susceptibles; además, se relaciona con cuencas hidrográficas que, ya de por sí, son susceptibles al cambio, tienen presión constante y estrés hídrico (Aguilar, 2009; SEMARNATCONAGUA, 2015). Al respecto, de las 13 ciudades grandes (mayores a un millón de habitantes), ocho se encuentran en el centro del país con aproximadamente 46 millones de habitantes y forman parte de las regiones hidrológicas Lerma-Santiago, Pánuco y Balsas, las cuales son catalogadas por su incidencia de riesgos y desastres asociados con fenómenos hidrometeorológicos y geológicos (Abeldaño \& González, 2018; SEDATU \& CONAPO, 2018).

Entre esas ciudades destaca Querétaro (ciudad conurbada por los municipios de Querétaro, Corregidora, El Marqués y Huimilpan), la cual presentó un significativo crecimiento poblacional y de superficie construida en los últimos 30 años; en 1990 tenía 537.100 habitantes con una superficie de $101,53 \mathrm{~km}^{2}$ y en 2020 contaba con 1.530 .820 habitantes y $205 \mathrm{~km}^{2}$ (último conteo de población y vivienda) (Delgado, 1993; SEDESOL \& CONAPO, 2012; INEGI, 2020). El aumento poblacional y físico de la ciudad está acompañado de su ubicación económico-estratégica entre el sur y norte del país, por la cercanía con la ciudad de México, el crecimiento industrial y el intenso desarrollo inmobiliario, por lo mismo, los cambios de uso de suelo y territoriales se han acentuado en un lapso relativamente corto (Arvizu, 2005; Hernández \& Osorno, 2018).

Los cambios señalados y de mayor repercusión en la ciudad de Querétaro se manifiestan en las subcuencas ${ }^{3}$ de la periferia urbana: en el norte Buenavista, Santa Rosa Jáuregui, Tierra Blanca, El Nabo y San José El Alto; al oeste se encuentra Tlacote El Bajo y El Pueblito, al sur están Joaquín Herrera y Los Olvera, y al este se presenta La Cañada y Ex Hacienda Menchaca, aunque cabe decir que algunas secciones de la periferia oeste y

3 La subcuenca o microcuenca son divisiones convencionales biofísicas hechas con fines de manejo de una macrocuenca, en México esa división también se manifiesta en cuencas ( $>50000$ hectáreas), subcuenca (5000 a 50000 hectáreas) y microcuenca (5000 hectáreas) (Cotler, 2010; IMTA, 2019). 
sur pertenecen a la cuenca central de Santiago de Querétaro (Hernández \& Martínez, 2017; Ochoa, 2019). Esos cambios se conducen con escasa vigilancia, diferenciación en la dotación de servicios e infraestructura urbana, especulación del suelo, limitada planeación, presión inmobiliaria, fragmentación territorial y cambios culturales de los pobladores originarios (Hernández \& Martínez, 2017). Además, se identifican problemas como la degradación de ecosistemas, reducción del arbolado y áreas verdes, ocupación de cauces y cuerpos de agua y transformación de suelos agrícolas por urbanos, todo lo anterior pareciera fomentar la construcción del riesgo de inundaciones (PNUMA, 2008; Peña, 2018).

En el periodo de 2000 al 2013 se registraron 69 inundaciones con daños y pérdidas materiales en el estado de Querétaro, mientras que en la ciudad de Querétaro fue de 43 eventos con esa magnitud (Hernández, 2013). Por su parte, solo en el área urbana del municipio de Querétaro se han identificado 31 sitios susceptibles por desbordamiento de cauces o zonas bajas de anegación (UAQ, 2009; UMPCQ, 2015). Sin embargo, en la periferia de la ciudad no existen registros claros de inundaciones, aunque algunos trabajos distinguen su formación en dos direcciones, el norte y el sur, donde el norte pareciera destacar en recurrencia y peligrosidad (UMPCQ, 2015; García \& Hernández, 2015; Peña, 2018).

En el sector norte destaca la subcuenca Santa Rosa Jáuregui, donde año con año se forman inundaciones con cada vez más afectaciones y aunque no se cuenta con un registro óptimo de los eventos, la escasa información permite señalar que las inundaciones se concentran en la sección baja de la subcuenca (UMPCQ, 2015). Además, pareciera que el problema aumentó en esa sección conforme la ocupación y alteración de la dinámica hídrica cuenca arriba, por lo tanto, ¿cuál es la distribución espacial de inundaciones por zonas funcionales en la subcuenca?, y, ¿cuáles son los factores que condicionan la formación de inundaciones por zonas funcionales?

Por lo comentado hasta el momento, el objetivo del presente trabajo fue analizar la formación de inundaciones por zonas funcionales en la subcuenca Santa Rosa Jáuregui en Querétaro. La respuesta a ese objetivo incluyó la identificación de zonas funcionales y un análisis hidrográfico, geológico y geomorfológico para determinar la susceptibilidad del lugar. El análisis permitirá tener un escenario de la formación de inundaciones 
donde se identifiquen problemas acumulativos y procesos escalonados que apoyen la toma de decisiones en la gestión y reducción del riesgo.

\section{Área de estudio}

La subcuenca Santa Rosa Jáuregui (SCSRJ) se localiza en la zona norte de la periferia urbana de la ciudad de Querétaro (Figura 1). Tiene una superficie de $100 \mathrm{~km}^{2}$ donde se establecen nueve localidades urbanas con 99.221 habitantes y 39 localidades rurales con 29.788 habitantes (INEGI, 2020). En la SCSRJ destacan tres lugares que pasaron de ser poblados antiguos a ser parte de las localidades urbanas de la ciudad de Querétaro, en orden de norte a sur son Santa Rosa Jáuregui, Juriquilla y Jurica. Los asentamientos poblacionales están distribuidos entre pendientes de $3^{\circ}$ a $25^{\circ} \mathrm{y}$ altitudes entre 1.980 y $2.450 \mathrm{msnm}$.

Figura 1. Localización de la subcuenca Santa Rosa Jáuregui

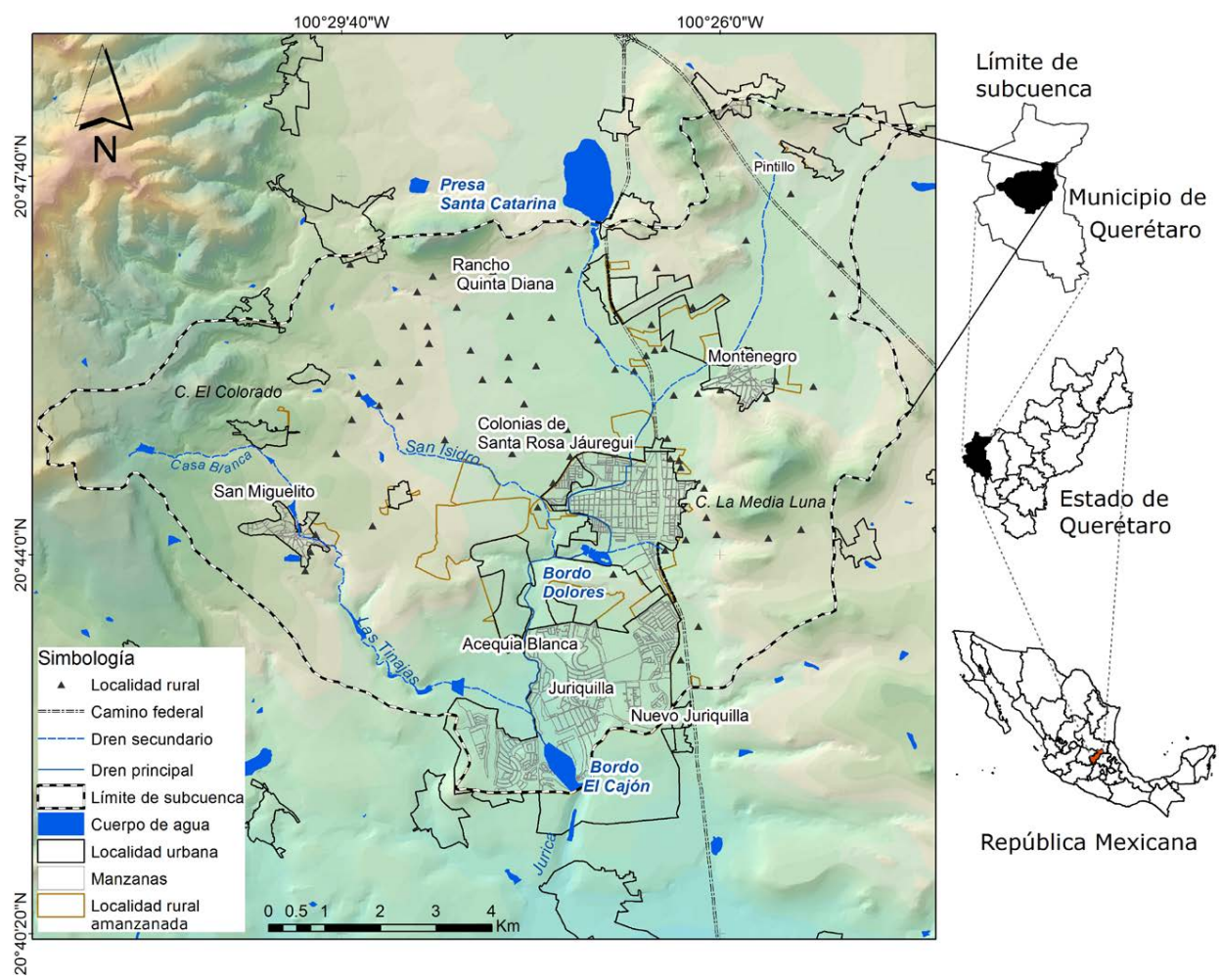

Fuente: Elaboración con información del INEGI (2015). 
El clima característico en la subcuenca es semiseco, donde la temperatura mínima es de $9^{\circ} \mathrm{C}$ y la máxima de $31,5^{\circ} \mathrm{C}$, mientras que la lluvia media anual es de $550 \mathrm{~mm}$. En los episodios de lluvia se tiene registro de la altura de agua entre $5 \mathrm{~cm}$ y $35 \mathrm{~cm}$, pero se han identificado registros de hasta 100 $\mathrm{cm}$ en algunas áreas bajas y limítrofes al Dren en Santa Rosa Jáuregui y en Jurica (UAQ, 2009). Asimismo, la SCSRJ está constituida por ocho diferentes usos de suelo, agricultura de temporal $(47,8 \%)$, zona urbana $(21,7 \%)$, matorral subtropical $(10,4 \%)$, matorral crasicaule $(7,5 \%)$, bosque tropical caducifolio $(5,0 \%)$, agricultura de riego $(4,3 \%)$, matorral espinoso $(2,0 \%)$ y pastizal inducido $(1,2 \%)$. Por su parte, desde la mitad y hasta el punto de salida de la SCSRJ, el predominio en el uso de suelo es agrícola y urbano, este último destaca por ser de condiciones mixtas (zonas habitacionales, comerciales y servicios) (Centro Queretano de Recursos Naturales, 2002).

La SCSRJ se encuentra en la Región Hidrológica 12 Lerma-Santiago, en ella se forma la cuenca Lerma-Chapala, siendo el río Querétaro una de sus principales vertientes. El agua que recibe ese río proviene del Valle de Buenavista y se encuentra controlada por la presa Santa Catarina, localizada al norte de la localidad Santa Rosa Jáuregui. En la vertiente oeste de Santa Rosa Jáuregui pasa el Dren del mismo nombre y llega al Bordo Dolores en la cabecera de la localidad, el cual forma parte de un parque de atracciones. El agua de esa presa sale regulada y direccionada a través de un Dren que, aguas abajo, se alimenta del arroyo San Isidro y cambia su nombre a Dren Jurica, este último desemboca en el Bordo El Cajón, también conocido como Lago de Juriquilla o club náutico, después de ello el Dren Jurica sigue su curso hasta desembocar en el río Querétaro.

En los parámetros morfométricos de la SCSRJ el índice de Gravelius fue 1,39 y el factor de forma 0,72 ; ello indica que la subcuenca es redonda a oval-oblonga, caracterizada por las crecidas de flujo de agua que tardan en llegar al punto de salida de la subcuenca (Bordo El Cajón). La red hídrica presenta una jerarquía de cuarto orden y la bifurcación de 2,0 que establece un menor grado de desarrollo fluvial y un potencial bajo de aguas de descarga hacia las secciones más bajas de la subcuenca. Por su parte, el terreno tiende a ser accidentado con una pendiente de $9,25 \%$ que, con la baja densidad de drenaje de $1,44 \mathrm{~km} / \mathrm{km}^{2}$, el flujo de agua recorre la superficie de manera lenta, con lo cual se incrementa el tiempo de concentración y disminuye el pico de crecida al presentar mayor infiltración. 


\section{Métodos y herramientas}

El objetivo del presente trabajo se responde con datos primarios de recorridos exploratorios, análisis hidrogeomorfológico y trabajo hemerográfico. Asimismo, para el procesamiento de información se utilizó un sistema de información geográfica (SIG). El trabajo de campo se llevó a cabo en el periodo de 2018 al 2020.

\section{Determinación de zonas funcionales}

Las zonas funcionales se fundamentan en la dinámica geomorfológica e hídrica de una cuenca, las cuales se asocian con la captación de agua de lluvia, almacenamiento, transporte, descarga y captación de agua (Valdés \& Hernández, 2018). Así, las zonas funcionales se determinaron con la sobreposición de información biofísica con base en Garrido et al. (2009), Valdés y Hernández (2018) y de Flotemersch et al. (2016).

En la primera parte del proceso se llevó a cabo la recopilación y el tratamiento de las capas biofísicas en formato vectorial. La capa de uso de suelo se elaboró con una técnica estándar de clasificación no supervisada en el programa ArcGis, para ello se utilizó de base el trabajo de Miranda (2007) y la capa de la serie VI del INEGI (2017), después se digitalizaron algunos rasgos distintivos al año 2020 obtenidos de la plataforma Google Earth Pro. La litología se obtuvo del Servicio Geológico Mexicano (2018) y fue tratada a partir de los aportes de Aguirre-Díaz, Zúñiga-Dávila, Pacheco-Alvarado, Guzmán-Speziale y Nieto-Obregón, (2000) y Xu, Nieto-Samaniego, Alaniz-Álvarez y Cerca-Martínez (2011). La hidrografía se obtuvo del INEGI (2010) y fue rectificada con base en el análisis de Flotemersch et al. (2016) y Valdés y Hernández (2018). La edafología se obtuvo de la capa INEGI (1973) y fue actualizada con el trabajo del Centro Queretano de Recursos Naturales (2002). A partir del modelo digital de elevación de la zona de estudio [curvas de nivel base equidistantes a 10 m del INEGI (2015)], se diseñó la geomorfología con base en los aportes de Bishop, James, Shroder y Walsh, (2011) y Hernández et al. (2017), y también se definió la capa del sistema de drenaje, donde su tratamiento fue a partir de los aportes de Reid (1993), Rosgen (1994) y Opere (2013).

Una vez obtenidas las capas con información biofísica, se integraron al SIG [(programa ArcGis (ESRI, 2013)], donde se transformaron las capas de formato vectorial a formato raster con celdas de 10 x $10 \mathrm{~m}$. Después, 
cada capa fue normalizada en cinco clases y se aplicó una suma lineal mediante la sobreposición de las capas a través del módulo raster calculator. El resultado de ese proceso fue la obtención de una sola capa, en la cual se analizaron y digitalizaron los límites de las zonas funcionales. Es importante decir que el proceso fue acompañado de dos salidas de trabajo de campo para corroborar o rectificar los resultados in situ.

\section{Unidades y secciones hidrogeomorfológicas}

Las unidades hidrogeomorfológicas consideran la relación entre la regulación hidrológica y geomorfológica (DIREN-PACA, 2007) y forman parte de la funcionalidad de la cuenca (Flotemersch et al., 2016). El proceso para determinar las unidades fue en dos fases. En la primera fase se consideró la delimitación cartográfica de la geomorfología fluvial para definir los accidentes geográficos en el relieve por el desarrollo del río principal de la subcuenca, se identificó la geomorfología litológica para considerar el relieve desde el tipo de roca aflorante y también se obtuvo la geomorfología estructural que da pauta al relieve resultante de una actividad tectónica (Aguirre-Díaz et al., 2000; Xu et al., 2011; DIREN-PACA, 2007). El proceso de esta primera fase fue elaborado sobre la plataforma de ArcGis, Google Earth Pro y recorridos en campo. En la segunda fase se asignaron valores de porosidad y conductividad hidráulica para definir los niveles de permeabilidad respecto a la litología presente en la subcuenca, para ello se tomaron en cuenta los trabajos del Instituto Tecnológico y Geominero de España (1987) y Langmuir (1997), así como los valores de conductividad hidráulica definida en el estudio de Morales (2011) para determinar zonas de recarga para el acuífero en la zona, además, esos mismos datos fueron extrapolados para las unidades litológicas definidas por Aguirre-Díaz et al. (2000), Xu et al. (2011) y el Servicio Geológico Mexicano (2018). La información de esta segunda fase fue procesada en el programa ArcGis; se utilizaron de base la cartografía geológica y los afloramientos identificados en el trabajo de campo.

Una vez integradas las dos fases, se realizaron tres secciones con el objeto de exponer el comportamiento hidrogeomorfológico e ilustrar zonas propensas a inundaciones de cada zona funcional: alta, media y baja. Sin embargo, el comportamiento del flujo de agua durante las precipitaciones estuvo en función del uso de suelo de la subcuenca. 


\section{Sitios susceptibles a inundaciones}

A raíz de los escasos registros sobre zonas de inundaciones fue necesario recopilar información a través de fuentes impresas y digitales. Así, durante el segundo semestre del 2019 se revisaron noticias sobre inundaciones publicadas en los periódicos locales que, para facilitar el proceso de búsqueda, se centró en todos los días del temporal de lluvias (mayo-octubre) en un periodo de años de 1999 a 2019. Después se revisaron registros de inundaciones establecidos en el atlas de riesgos del municipio de Querétaro (UMPCQ, 2015), el plan maestro pluvial (UAQ, 2009), el programa estratégico para temporada de lluvias (UMPCQ, 2017), el programa estatal de acción ante el cambio climático (Hernández, 2013) y el atlas nacional de riesgos CENAPRED (2019). También se revisaron publicaciones sobre inundaciones, sean tesis de posgrado (Michel, 2016; Peña, 2018) o artículos (García \& Hernández, 2015; González, 2018; Michel \& Hernández, 2019).

Con la información recabada se elaboró una base de datos constituida por el número de registro, coordenadas o elementos de ubicación, frecuencia de registro, atención brindada y posibles pérdidas económicas y humanas. La base de datos incluyó sitios y áreas de inundación, en ese sentido, la base de datos se dividió en puntos y polígonos para ser incluidos en el SIG y adicionar las zonas funcionales y facilitar la producción cartográfica.

\section{Registros de precipitación pluvial}

De manera adicional a la información documental y zonas funcionales fue necesario incluir los datos de precipitación, ya que es una de las variables importantes en el desarrollo de las inundaciones. Así, se utilizaron registros de precipitación mensual, anual y máxima en $24 \mathrm{~h}$ de la estación La Joya y la estación Juriquilla (CONAGUA, 2018), las cuales son las más cercanas a la subcuenca (menores a $8 \mathrm{~km}$ ), cuentan con periodos de registro de 30 años (1985-2015) y no presentan alteraciones topográficas de consideración entre ellas.

\section{Resultados y discusión}

Se definieron tres zonas funcionales para la SCSRJ: a) zona funcional alta (ZFA), sección de captación de agua con una superficie de 33,8 $\mathrm{km}^{2}$, pendiente promedio de $14 \% \mathrm{y}$ unidades geomorfológicas con predominio de lomeríos altos y medios; b) zona funcional media (ZFM), sección de almacenamiento y transporte de agua, tiene una superficie de $55 \mathrm{~km}^{2}$, 
pendiente promedio de $6 \%$ y unidades geomorfológicas con predominio de lomeríos medios y bajos; y c) zona funcional baja (ZFB), cuenta con una superficie de $11,2 \mathrm{~km}^{2}$, pendiente promedio de $3 \%$, en las unidades geomorfológicas predomina la llanura de inundación, seguida de lomeríos bajos y planicie. Cada zona funcional depende de las unidades hidrogeológicas determinadas por particularidades del comportamiento litológico y la conductividad hidráulica, por ello, la ZFA y ZFM están compuestas por unidades hidrogeológicas de media a alta y de baja a media permeabilidad, mientras que la ZFB está integrada por unidades hidrogeológicas con alta y media a alta permeabilidad (Figura 2).

Figura 2. Zonas funcionales y unidades hidrogeológicas.

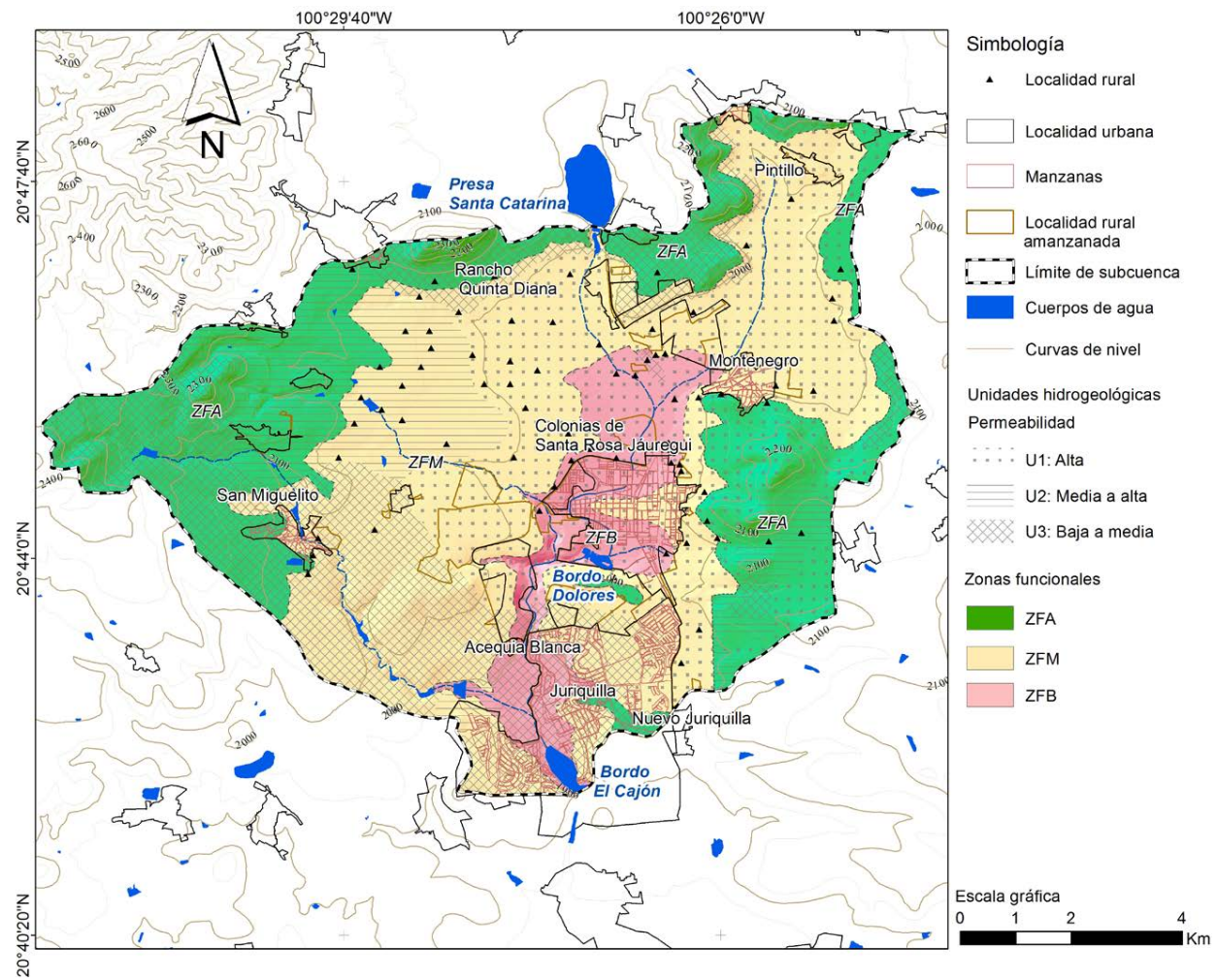

Fuente: Elaborado con datos de Aguirre-Díaz et al. (2000), Xu et al. (2011), Morales (2011) y SGM (2018). 
Cotler (2007) y Valdés y Hernández (2018) consideran que la estructura sistémica de la cuenca depende también de las subunidades que la integran, las cuales propician la respuesta de la cuenca con diferentes escenarios funcionales. Así, al relacionar la litología con las unidades hidrogeológicas destacan la ZFM y la ZFB con dos escenarios. En el primer escenario, la ZFM puede formar avenidas torrenciales que descargan en la planicie aluvial de la ZFB, la cual es susceptible a la formación de inundaciones. En el otro escenario, la ZFM y la ZFB presentan unidades hidrogeológicas U1, ello puede funcionar como captación de agua debido a los depósitos lacustres y fluviales que la integran (Tabla 1), eso facilitaría la conducción del agua hacia los niveles freáticos que, en dependencia del tipo de suelo, uso de suelo y la vegetación, pueden mantener las funciones de la subcuenca.

Tabla 1. Unidades hidrogeológicas de las zonas funcionales de la SCSRJ.

\begin{tabular}{|c|c|c|c|c|}
\hline Litología & Código & Área $\left(\mathbf{k m}^{2}\right)$ & Unidad hidrogeológica & Zona funcional \\
\hline Piroclásticos Ezequiel Montes & $\mathrm{TmTq}$ & 7,2 & \multirow[t]{2}{*}{ U1: permeabilidad alta } & ZFA, ZFM \\
\hline Depósito lacustre y fluvial & MQ & 37,5 & & ZFB, ZFA, ZFM \\
\hline Andesita El Cedro & ToA & 0,8 & \multirow{3}{*}{$\begin{array}{l}\text { U2: permeabilidad media } \\
\text { a alta }\end{array}$} & ZFA \\
\hline Basalto & $\mathrm{TmB}$ & 13,2 & & ZFA, ZFM \\
\hline Obrajuelo-Dacita & TDo & 2,6 & & ZFA \\
\hline Caliza y Lutita & Kic & 1,7 & \multirow{3}{*}{$\begin{array}{l}\text { U3: permeabilidad de baja } \\
\text { a media }\end{array}$} & ZFA, ZFM \\
\hline Andesita, Basalto & TmA-B & 8,8 & & ZFA \\
\hline Basalto Querétaro & TAB & 27,9 & & ZFB, ZFA, ZFM \\
\hline
\end{tabular}

Fuente: Elaborado con datos de Aguirre-Díaz et al. (2000), Xu et al. (2011), Morales (2011) y SGM (2018).

Las características edafológicas de mayor predominancia en el área de recarga de la ZFA es litosol y vertisol pélico más grava, equivalente al $75 \%$, con pendiente mayor de $8 \%$. En la ZFM, de transporte, el tipo de suelo es vertisol pélico más grava, representa el $89 \%$ y tiene una pendiente menor al 8\%. En la planicie aluvial de la ZFB el 80\% del área es de tipo vertisol pélico de textura fina con pendientes menores al 3\%. Opere (2013) menciona que, en algunos casos de lluvias intensas, las capas de arcilla se conducen por escorrentías superficiales o generan depósitos; cualesquiera la razón, forman inundaciones en periodo de tiempo relativamente corto, pero con tiempos prolongados para la salida o desecación del agua. En ese sentido, la ZFB presenta condiciones similares, pues el vertisol pélico 
contiene arcillas expandibles de baja infiltración que ocasionan inundaciones en periodos prolongados, mientras que en la ZFM se pueden formar escorrentías torrenciales de rápida respuesta.

Aunado a lo anterior, el cambio de uso de suelo y vegetación entre los años 2007 y 2020 ha intervenido en la formación de escorrentías torrenciales e inundaciones en la SCSRJ (Figura 3). En la ZFA no se presentaron importantes cambios de uso de suelo, predomina la vegetación de matorral y agricultura de temporal, eso incide de manera positiva en las funciones de la subcuenca. Por su parte, la ZFM sí presentó cambios significativos, aumentó en 4\% la superficie de agricultura de riego y en 7\% el uso urbano, esos cambios pueden alterar la velocidad de los escurrimientos torrenciales, arrastre de sedimento y acelera la formación de las inundaciones. En la ZFB predomina el uso de suelo urbano, el cual aumentó en 3\%, eso implica que el área de descarga y depósito de agua se sigue ocupando y modificando, por lo mismo, interviene no solo en la formación de inundaciones, sino también en la alteración de las subcuencas vecinas. Al respecto, Fu, Jang, Huang, Lin y Yeh (2018) mencionan la importancia de evaluar los cambios de usos de suelo y la cobertura de vegetación a escala de cuenca y cuencas vecinas como prevención de las inundaciones causadas por las escorrentías de la cuenca alta asociadas por las alteraciones de la urbanización.

Figura 3. Cambio de uso suelo y vegetación en zonas funcionales de la SCSRJ, 2007-2020.

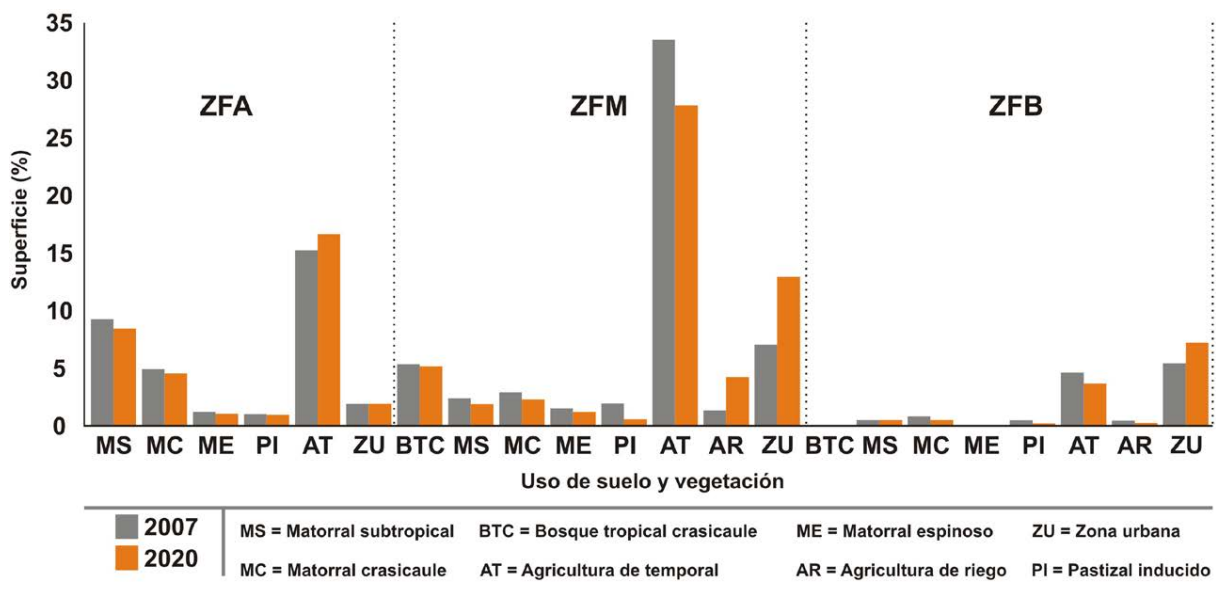

Fuente: Elaboración con base en Miranda (2007) y el INEGI (2017). 
Para la hidrogeomorfología, las tres secciones trasversales en la SCSRJ demostraron nuevamente que la estructura de la subcuenca incide de manera escalonada y finaliza con el depósito de agua sobre las llanuras de inundación y drenes de tercer y cuarto orden, los cuales se localizan sobre la ZFM y ZFB, que a la vez se encuentran constituidas por unidades hidrológicas U3 y U1 (Figura 4). En ambas zonas los subsuelos están representados por depósitos lacustre y fluvial (MQ) definidos como U1, esto es, zonas permeables (Sección 1 y 2). Sin embargo, las superficies urbanizadas llegan a funcionar como una gran capa impermeable que propicia la desviación transversal y horizontal del flujo hídrico con consecuencias escalonadas entre zonas funcionales. Eso confirma lo señalado por Forman (2008) y Weng, Ruhana, Zaini, Lee e Hin (2016), donde la urbanización conlleva coberturas del suelo impermeables que desvían los escurrimientos pluviales e impiden la infiltración del agua al manto freático, por esto, de manera escalonada se producen las afectaciones, tal como sucede entre la ZFM y ZFB.

Figura 4. Zonas de inundaciones en tres perfiles transversales de la SCSRJ
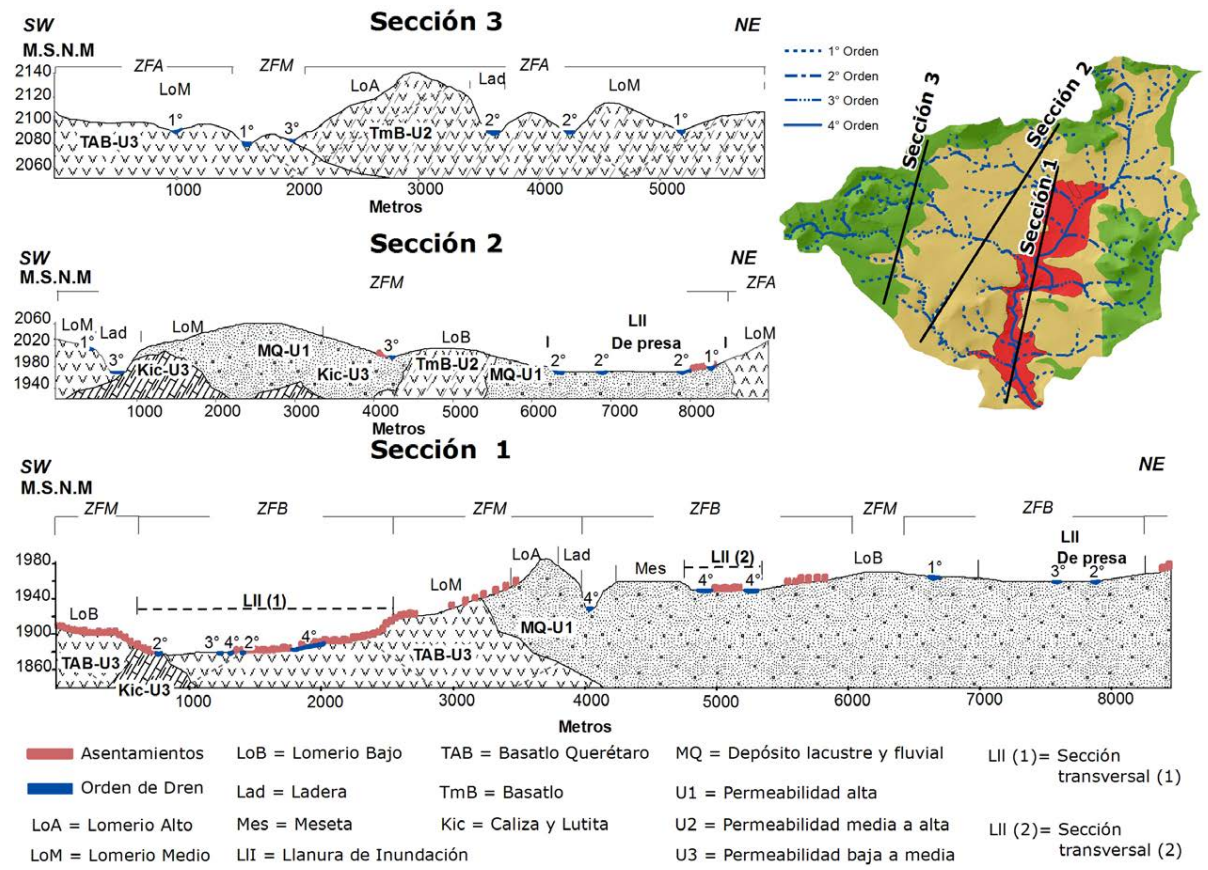

Fuente: Elaborado con datos de Aguirre-Díaz et al. (2000), Xu et al. (2011), Morales (2011) y SGM (2018). 
Respecto a los eventos de inundaciones, la recopilación hemerográfica y de documentos oficiales en el periodo de 1999 a 2019 da cuenta de un total de 234 eventos de inundaciones en el Estado (12 por año), donde el $62 \%$ del total se presentó en la ciudad de Querétaro. En cuanto a la SCSRJ, se tuvo un vacío de información en los cinco años iniciales de ese periodo, por lo cual, de 2006 al 2019 se presentaron un total de 69 eventos de inundaciones: ZFA con dos eventos, ZFM con 15 eventos y ZFB con 52 eventos, además, cabe decir que coinciden con los episodios de lluvia más altos (Figura 5).

Referente a los registros de precipitación pluvial, los meses de junio, julio y agosto son los de mayor precipitación, donde la precipitación máxima en $24 \mathrm{~h}$ oscila entre $45 \mathrm{~mm}$ y $61 \mathrm{~mm}$. Aunque cabe decir que en los primeros 35 minutos es cuando la lluvia presenta la mayor intensidad y suele formar inundaciones repentinas o relámpago. Al respecto, Hernández (2013) señala que las inundaciones con mayores afectaciones en la ciudad se produjeron por ese tipo de inundaciones, como sucedió en los años de 1998, 2001, 2003, 2005, 2007, 2013 y 2017; el último evento ocasionó una persona fallecida en la ZFB.

Figura 5. Precipitación máxima mensual e inundaciones por zonas funcionales de la SCSRJ.

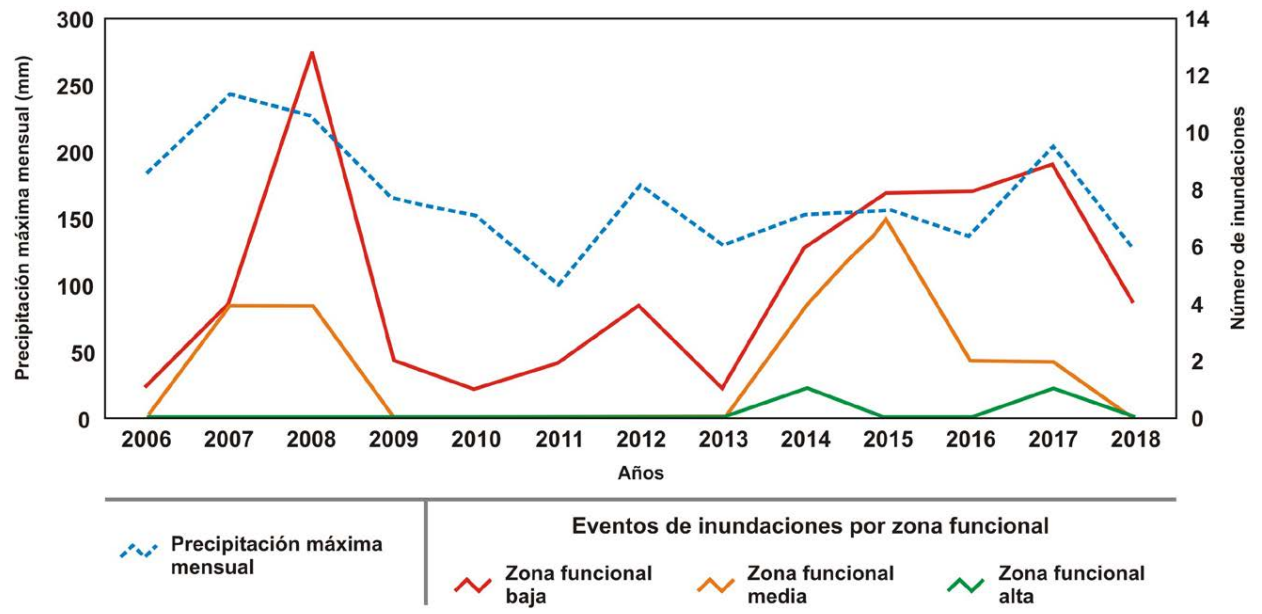

Fuente: Elaborado con la recopilación hemerográfica, documental y de la CONAGUA (2018). 
Con la información anterior, nuevamente las inundaciones recaen entre la ZFM y la ZFB, donde se obtuvo que los registros de la primera se asocian con la ocupación de drenes o puntos de concentración de agua donde se conjuntan drenes de tercer y cuarto orden, además, se relacionan directamente con los registros de precipitación (Figura 6). García y Hernández (2015) notificaron que en la administración municipal del periodo de 2009 a 2012 fueron atendidos algunos tramos del Dren de Santa Rosa Jáuregui y en las cercanías del Bordo Dolores, esa atención tuvo el objetivo de mitigar las inundaciones en la ZFB, pero desde el año 2013 incrementaron los registros. En este aspecto, las soluciones temporales no siempre logran mitigar, mientras que la formación y efectos de las inundaciones puede ser cíclico y acumulativo (Reid, 1993; Walker et al., 2016). Por otro lado, las inundaciones en la ZFB propiciaron importantes afectaciones en el periodo señalado, mucho se debe a la ya mencionada ocupación de sitios susceptibles, en especial, aquellos contiguos o sobre drenes (segundo, tercer y cuarto orden), depresiones y cauces temporales. Además, en la información obtenida resaltó de manera considerable la influencia del mal manejo de las presas reguladoras establecidas en las secciones de transición entre la ZFM y ZFB.

Figura 6. Incidencia de inundaciones en las zonas funcionales de la CSRJ

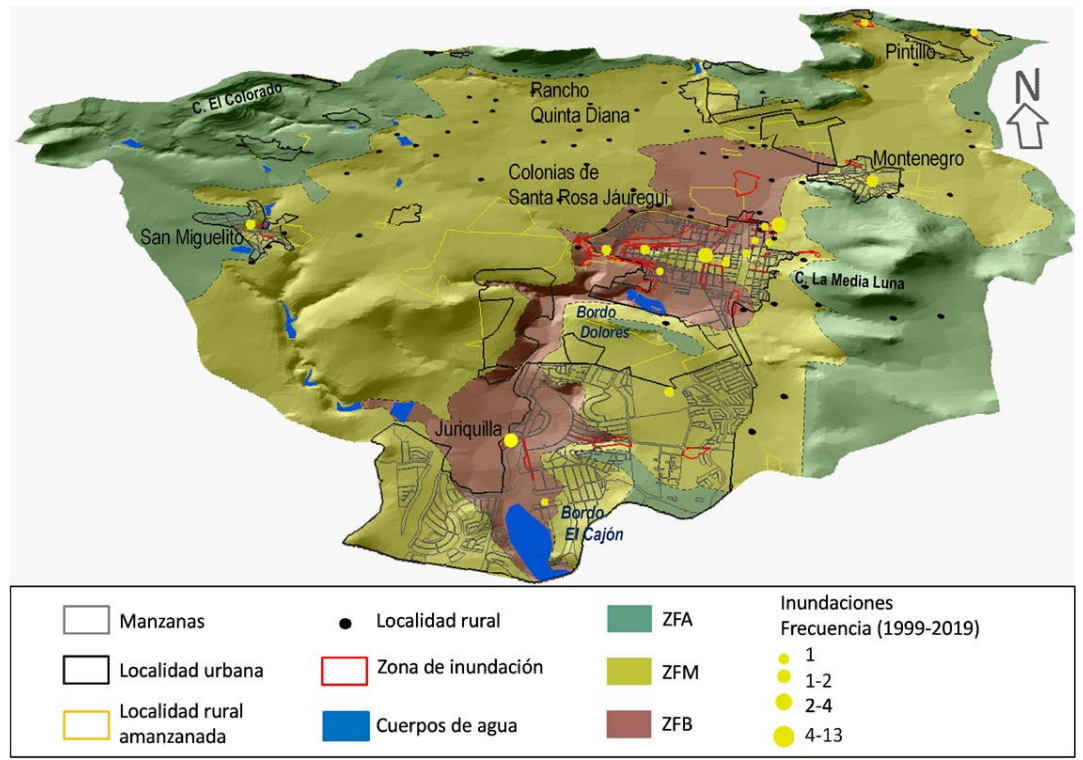

Fuente: Elaboración propia con base en datos hemerográficos, trabajo de campo y el INEGI (2015). 
Los sitios de inundaciones con cobertura urbana en las llanuras de inundaciones que se observan en la Figura 7 ocupan los drenes de cuarto orden en la ZFB [también ver la Figura 4, sección transversal LII (1) y sección transversal LII (2)] y son por naturaleza inundables. Lo anterior se sustenta por las condiciones de las rocas, suelo, sinuosidad, amplitud, profundidad, vegetación y forma de atrincherado, lo cual clasifica ríos con alta sensibilidad a las perturbaciones, buena potencia de recuperación, alto suministro de sedimentos, alto potencial de erosión en el cauce y alta influencia en el control de vegetación; todo ello con base en la clasificación de ríos de Rosgen (1994). Adicional a lo anterior, la composición de suelo (vertisol pélico) y roca (basaltos vesiculares) [Sección transversal LlI (1)], así como la alteración de la vegetación, permiten la formación de acumulación del agua con precipitaciones intensas y a las cuales los asentamientos susceptibles están expuestos.

Figura 7. Secciones transversales en los cauces de cuarto orden en las llanuras de inundaciones de la Sección transversal hidrogeomorfológica 1

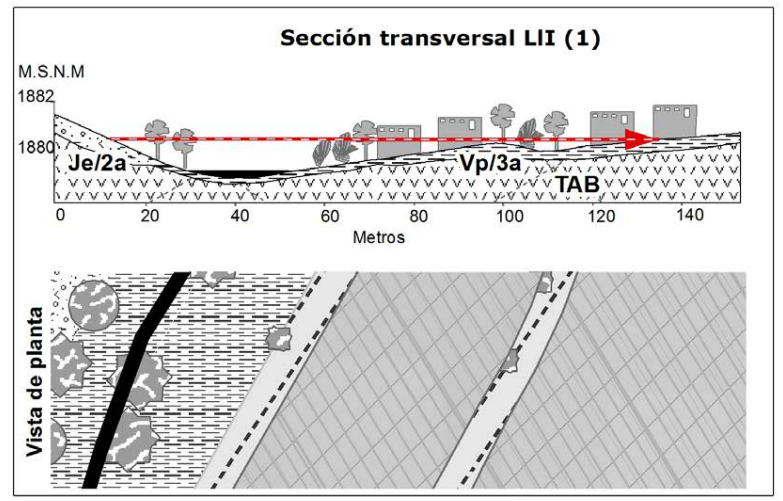

Fluvisol éutrico, textura media, pendiente menor a $3 \%(\mathrm{Je} / 2 \mathrm{a})$

Vertisol pélico, textura fina, pendiente menor a $3 \%(\mathrm{Vp} / 3 \mathrm{a})$
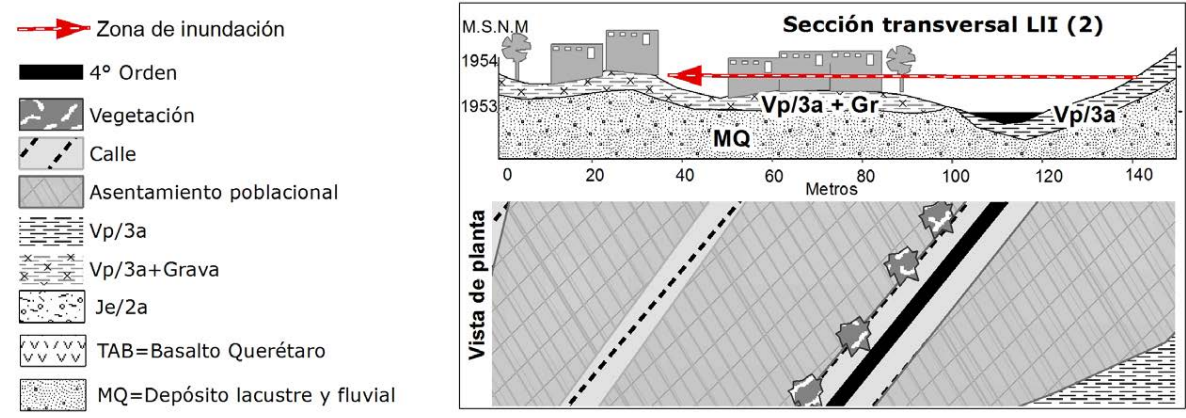

Fuente: Elaboración con base del INEGI (1973), Aguirre-Díaz et al. (2000), Xu et al. (2011) y SGM (2018). 
En la misma Figura 7 (vista de planta), se corrobora que la mayoría de las áreas susceptibles a inundaciones de la ZFM y ZFB están siendo ocupadas, pero esa ocupación debería estar 1.30 metros por encima del suelo en donde actualmente se asientan, sin embargo, a pesar de las limitadas acciones de infraestructura y vigilancia sobre la periferia urbana, esas zonas continuarán siendo prioritarias. Lo anterior podría cambiar al limitar la ocupación y, por ende, también conservar la estructura de la ZFA. También es importante comprender que, debido a la propia condición y actividades que se llevan a cabo en la periferia urbana, la infraestructura y los medios de mitigación deben ser distintos al área urbana central para mantener las funciones de la subcuenca (Gunnell et al., 2019).

A partir de los resultados encontrados se puede señalar que las inundaciones en la SCSRJ son producto de impactos acumulados entre zonas funcionales y no solo de un elemento en específico. Por ejemplo, si bien la lluvia ha sido un elemento clave en la formación de inundaciones, quedó de manifiesto que las alteraciones al lugar (geomorfológica, litológica, edáfica e hidrográfica) son variables determinantes, ya que modifica la estructura sistémica de las zonas funcionales con repercusiones recíprocas entre ellas, a la vez que puede afectar subcuencas vecinas. Esto sustenta lo mencionado por Walker et al. (2016), donde el impacto que sufren las subunidades de una cuenca produce alteraciones estructurales y sistémicas que reducen la efectividad de la conectividad entre zonas funcionales, mientras que Gunnell et al. (2019) y Mavhura (2019), mencionan que la precipitación acompañada de cualquier alteración al lugar incide en el riesgo de formar inundaciones y complejiza los mecanismos de mitigación.

Es importante señalar que la rapidez con la que se ocupan sitios susceptibles es inversamente proporcional a la capacidad del sistema para mantener su funcionalidad como lo mencionan Hernández et al. (2012) y Jha, Bloch y Lamond, (2012). Por esta razón, la ZFA todavía presenta cierta estructura de óptimo desempeño sistémico y la formación de inundaciones es casi nula. Sin embargo, las alteraciones hidrogeológicas e hidrogeomorfológicas en la ZFM se relacionan en las inundaciones de esa zona y en la formación de inundaciones de la ZFB. García y Hernández (2015) mencionan que las acciones para mitigar inundaciones en la ZFB han sido encausar los escurrimientos y brindar limpieza antes del temporal de lluvias, pero valdría la pena revisar la situación de la ZFA y ZFM para realizar acciones con mayores 
argumentos que fortalezcan la gestión del riesgo. Sin embargo, de continuar los cambios de uso de suelo a la velocidad actual, en el mediano plazo se verá afectada la ZFA, y con ello toda la subcuenca, lo que complicará aún más las medidas de mitigación o solución.

\section{Conclusiones}

En el presente trabajo se definieron y analizaron tres zonas funcionales para la subcuenca Santa Rosa Jáuregui. La zona funcional alta se considera de recarga de agua y está compuesta de lomeríos medios y altos con escasas alteraciones en su estructura y función. La zona funcional media se caracteriza por el transporte de agua y está representada por lomeríos bajos con alteraciones a causa del cambio de uso de suelo de agrícola a urbano. Por su parte, la zona funcional baja es de acumulación de agua y presentó alteraciones hidrogeomorfológicas asociadas al uso urbano sobre la llanura de inundaciones.

La formación de inundaciones en la subcuenca coincide con las modificaciones propiciadas por la cobertura urbana entre la zona funcional media y la zona funcional baja. La zona funcional media, producto de la cobertura urbana, transformó la permeabilidad del suelo por secciones impermeables que, dada la fragilidad hidrogeológica, favorece avenidas torrenciales hasta la llanura de inundaciones, esto es, en la zona funcional baja, en la cual el agua se retiene, se desvía a través de canales o se ralentiza su salida de la subcuenca.

Así, cualquier alteración que se realice en alguna de las zonas funcionales repercute en todo el sistema. Por lo tanto, en la subcuenca Santa Rosa Jáuregui la zona funcional alta debe conservar sus condiciones actuales, mientras que la zona funcional media debe aminorar las perturbaciones producto del cambio de uso de suelo que modifica la dinámica hídrica y que repercute en la siguiente zona, por lo cual, la zona funcional baja requiere limitar la velocidad de los cambios de uso de suelo y presentar mejores estrategias de planeamiento para mitigar inundaciones, sean de forma sistémica, vertical u horizontal.

Los resultados expuestos pueden ser considerados en planes, programas, reglamentos y normativas para atender de forma integral aquellos elementos perturbadores que fomentan la construcción del riesgo y atender los sectores más susceptibles, tal como lo señalan las metas de los objetivos de desarrollo sostenibles para el año 2030 y los esfuerzos de ONUHábitat por promover ciudades sustentables. 


\section{Agradecimientos}

Los autores agradecen el financiamiento a través del proyecto Estrategias de respuesta y adaptación a riesgos socio-naturales en la periferia urbana de la ciudad de Querétaro, México (UAQ-FNB202009), al proyecto Nuevas geografías de la urbanización en México: Transformaciones territoriales y medios de vida de sectores sociales vulnerables en las periferias de ciudades medias (PAPIIT-UNAM) y al Consejo Nacional de Ciencia y Tecnología (CONACYT).

\section{Referencias}

Abeldaño, R.\& González, A. (2018). Desastres en México de 1900 a 2016: patrones de ocurrencia, población afectada y daños económicos. Revista Panamericana de Salud Pública, 42, 1-8. doi: https://doi. org/10.26633/RPSP.2018.55

Aguilar, G. (2009). Urbanización periférica e impacto ambiental. El Suelo de conservación en la Ciudad de México. En: G. Aguilar e I. Escamilla. (Eds.), Periferia Urbana: Deterioro ambiental y reestructuración metropolitana (pp. 21-52). México, D.F.: Miguel Ángel Porrúa. Aguirre-Díaz, G., Zúñiga-Dávila, F., Pacheco-Alvarado, F., GuzmánSpeziale, M., \& Nieto-Obregón, J. (2000). El graben de Querétaro, México. Observaciones de fallamiento activo. GEOS, 20(1), 2-7.

Arroyo, L. (2018). Riesgos de desastres en áreas periurbanas en la gran área metropolitana, consideraciones pasadas y recientes: orientaciones y alcances reales de la gestión del riesgo y el ordenamiento territorial, cantones de Aserrí, Alajuelita, Desamparados, Escazú, Santa Ana y Mora. Revista Geográfica de América Central, 1(60), 231254. doi: 10.15359/rgac.60-1.8

Arvizu, C. (2005). Evolución urbana de Querétaro (1531-2005). Querétaro: Municipio de Querétaro/Tecnológico de Monterrey.

Barbedo, J., Miguez, M., Van der Horst, D., \&Marins, M. (2014). Enhancing ecosystem services for flood mitigation: a conservation strategy for peri-urban landscapes? Ecology and Society, 19(2), 54.doi: https://doi.org/10.5751/ES-06482-190254

Becerra, S., Peltier, A., Antoine, J., Labat, D., Chorda, J., Ribolzi, O., Daupras, F. \& Dartus, et D. (2013). Comprendre les comportements face à un risque modéré d'inondation. Etude de cas dans le périurbain 
toulousain (Sud-Ouest de la France), Hydrological Sciences Journal, 58(5), 945-965. doi: 10.1080/02626667.2013.786181

Bishop, M. P., James, L. A., Shroder, J. F.\& Walsh, S. J. (2011). Geospatial technologies and digital geomorphological mapping: Concepts, issues and research. Geomorphology, 137(1), 5-25. doi: https://doi. org/10.1016/j.geomorph.2011.06.027

Braud, T., Fletcher, T. \& Andrieu, H. (2013). Hydrology of peri-urban catchments: process and modelling. Journal of Hydrology, 485, 1-13. https://hal.archives-ouvertes.fr/hal-00916031/document

Califano, F., Mobilia, M.\& Longobardi, A. (2015). Heavy rainfall temporal characterization in the peri-urban Solofrana river basin, Southern Italy. Procedia Engineering, 119, 1129-1138. doi: https://doi. org/10.1016/j.proeng.2015.08.957

Cardoso, M. M. \& Fritschy, B. A. (2012). Revisión de la definición del espacio rururbano y sus criterios de delimitación. Contribuciones cientificas GAEA, 24, 27-39.

Centro Nacional de Prevención de Desastres (CENAPRED). (2019). Atlas Nacional de Riesgos. Ciudad de México: CENAPRED. Recuperado de: http://www.atlasnacionalderiesgos.gob.mx/

Centro Queretano de Recursos Naturales. (2002). Uso actual y potencial del suelo en los municipios conurbados de Querétaro. Querétaro: CONCYTEQ.

Comisión Nacional del Agua (CONAGUA). (2018). Red de estaciones climatológicas. Ciudad de México: Servicio Meteorológico Nacional. Recuperado de https://smn.conagua.gob.mx/es/climatologia/ informacion-climatologica/informacion-estadistica-climatologica

Cotler, H. (2007). El manejo integral de cuencas en México. Ciudad de México, México: SEMARNAT. Recuperado de: https://agua.org. mx/wp-content/uploads/2008/06/El-Manejo-Integral-de-Cuencasen-Mexico-segunda-edici\%C3\%B3n.pdf

Cotler, H. (Coord.) (2010). Las cuencas hidrográficas de México. Diagnóstico y priorización. Ciudad de México: SEMARNAT, INE, FGRA.

Craig, D.\& Jeffery, M. (2014). Adaptive governance for exreme events in peri-urban areas: a case study of greater western Sydney. En: B. Maheshwari, R. Purohit, H. Malano, V. Singh\& P. Amerasinghe. (Eds.). The security of water, food, energy and liveability of cities. 
Challenges and opportunities for peri-urban futures (pp. 449-462). Texas: Springer.

Cutter, S., Boruff, B. \& Lynn, W. (2003). Social vulnerability to environmental hazards. Social Science Quarterly, 84(2), 424-261. doi: https://doi.org/10.1111/1540-6237.8402002

Delgado, J. (1993). Querétaro: hacia una ciudad-región: Estudios demográficos y urbanos, 8(3), 655-699. doi: 10.24201/edu.v8i3.889

Direction Régional de I'Environmnet-Provence Alpes Cote D'Azur (DIREN-PACA). (2007). L'approche hydrogéomorphologique en milieux méditerranéens. Une méthode de détermination des zones inondables. Recuperado de http://www.paca.developpement-durable.gouv.fr/ IMG/pdf/L_approche_hydrogeomorphologique_cle71a4d9.pdf

Eakin, H., Lemer, A. y Murtinho, F. (2010). Adaptive capacity in envolving peri-urban spaces: Responses to flood risk in the upper Lerma River Valley, Mexico. Global Environmental Change, 20(1), 14-22. doi: https://doi.org/10.1016/j.gloenvcha.2009.08.005

Economic and Social Research Institute (ESRI). (2013). Software ArcGis, version 10.0, Nueva York: ESRI.

Flotemersch, J. E., Leibowitz, S. G., Hill, R. A., Stoddard J. L., Thoms, M. C., \& Tharme, R. E. (2016). A watershed integrity definition and assessment approach to support strategic management of watersheds. River Research and Applications, 32(7), 1654-1671. doi: https://doi. org/10.1002/rra.2978

Forman, R. T. (2008). Urban Regions Ecology and Planning Beyond the City. New York, United States: Cambridge University.

Fu J., Jang, J., Huang, C., Lin, W., \& Yeh, C. (2018). Cross-analysis of land and runoff variations in response to urbanization on basin, watershed, and city scales with/without green infrastructures. Water, 10(2), 106. doi: https://doi.org/10.3390/w10020106

García, L., \& Hernández, J. (2015). Capacidades de respuesta de los habitantes de Santa Rosa Jáuregui al riesgo asociado a inundaciones en la ciudad de Querétaro, México. Revista Digital CIENCIA@UAQ, $8(2), 1-17$.

Garrido, A., Enríquez, C., Pérez, J., Luna, N., \& Sánchez, O. (2009). Zonas funcionales de las cuencas hidrográficas de México. Escala 1:250000. D.F. México: INECC. 
González, E. (2018). Propensión de las inundaciones históricas de los últimos 100 años en Querétaro. Revista Digital CIENCIA@UAQ,11(1), 178-192.

Gunnell, K., Mulligan, M., Francis, R., \& Hole, D. G. (2019). Evaluating natural infrastructure for flood management within the watersheds of selected global cities. Science of the Total Environment, 670, 411424. doi: https://doi.org/10.1016/j.scitotenv.2019.03.212

Hernández, G. (2013). Diagnóstico de escenarios de riesgo vinculado con eventos y amenazas de origen hidrometeorológico en el estado de Querétaro. En: H. Suzán, V. Cambrón, O. García, A. Guevara, H. Luna., \& E. González. (Eds.). Programa estatal de acción ante el cambio climático-Querétaro (pp. 145-169). Querétaro: SEMARNAT, INECC, SEMARNAT.

Hernández, G. J., Vieyra, M. A., \& Mendoza, M. E. (2012). Adaptation strategies in communities under precarious housing: Flooding risks in the peri -urban sector of the city of Morelia, Michoacán, México. Applied Geography, 34, 669-679. doi: https://doi.org/10.1016/j. apgeog.2012.04.010

Hernández, J. R., Pérez, J. L., Rosete, F., Villalobos, M., Méndez, A. P., \& Navarro, E. (2017). Clasificación geomorfológica del relieve mexicano: una aproximación morfográfica por densidad de curvas de nivel y la energía del relieve. Investigaciones Geográficas. Instituto de Geografía, UNAM, (94), 1-15. doi: 10.14350/rig.57019

Herrnández, J., \& Martínez, G. (2017). Gestión local de cuerpos de agua en la microcuenca San José El Alto ante la dinámica urbana de la ciudad de Querétaro. En: M. Ribeiro, y E. Belmont. (Coords.). Problemas emergentes en ciudades medias (pp. 81-102). Ciudad de México: Plaza y Valdés S.A. de C.V.

Hernández, J., \& Osorno, T. (2018). Diferencias ambientales en el paisaje urbano de la ciudad de Querétaro, México: caso de estudio Juriquilla y Santa Rosa Jáuregui. Revista de Geografia Norte Grande, (71), 147-166. doi: https://doi.org/10.4067/S0718-34022018000300147

Instituto Mexicano de Tecnología del Agua (IMTA). (2019). ¿Qué es una cuenca? Recuperado de: https:/www.gob.mx/imta/articulos/ que-es-una-cuenca-211369\# 
Instituto Nacional de Estadística y Geografía (INEGI). (1973). Carta edafológica. Querétaro. Escala 1:50000. Recuperado de: https://www. inegi.org.mx/temas/edafologia/

Instituto Nacional de Estadística y Geografía (INEGI). (2010). Red hidrográfica. Subcuenca hidrográfica RH12Hd Río Apaseo, Cuenca Rio Laja. RH Lerma-Santiago, 1:50000. Recuperado de: https://www. inegi.org.mx/temas/hidrografia/default.html\#Descargas

Instituto Nacional de Estadística y Geografía (INEGI). (2015). Conjunto de datos vectoriales y toponímicos, escala 1:50000, claves F14C55 y F14C65. Recuperado de: https://www.inegi.org.mx/app/biblioteca/ficha.html?upc $=702825270896$

Instituto Nacional de Estadística y Geografía (INEGI). (2017). Conjunto de datos vectoriales de la carta de uso del suelo y vegetación. Escala 1:250000. Serie VI. Recuperado de: https://www.inegi.org.mx/app/ biblioteca/ficha.html?upc $=889463598459$

Instituto Nacional de Estadística y Geografía (INEGI). (2020). Censo de Población y Vivienda 2020. Recuperado de: https://www.inegi.org. $\mathrm{mx} /$ programas/ccpv/2020/

Instituto Tecnológico y Geominero de España. (1987). Manual de Ingeniería de Taludes. Madrid: Instituto Tecnológico Geominero de España. Jáuregui, E., Aversa, M., \& Salas, R. (2016). Estrategias para la mitigación del riesgo por inundación: caso cuenca del arroyo Maldonado, La Plata, Buenos Aires, Argentina. URBANO, 19(34), 34-47. doi: https://doi.org/10.22320/07183607.2016.19.34.4

Jha, A. K., Bloch, R., \& Lamond, J. (2012). Cities and flooding. A guide to Integrated Urban flood risk management for the 21st Century. Washington, DC: The World Bank.

Langmuir, D. M. (1997). Aqueous Environmental Geoquemistry. EUA: Prentice Hall.

Mavhura, M. (2019). Analysis drivers of vulnerability to flooding: a systems approach. South African Geographical Journal, 101(1), 72-90. doi: https://doi.org/10.1080/03736245.2018.1541020

Michel, A. (2016). Riesgo por escurrimiento hídrico superficial y propuesta de mitigación en La Cañada Menchaca, Querétaro. Tesis de maestría. Universidad Autónoma de Querétaro. México. 
Michel, A., \& Hernández, J. (2019). Vulnerabilidad ante la ocurrencia de avenidas torrenciales en la microcuenca Menchaca, Querétaro. Revista NTHE, (27), 20-26.

Miller, J., Kim, H., Kjeldsen, T., Packman, J., Grebby, S., \& Dearden, R. (2014). Assesing the impact of urbanization on storm runoff in a peri-urban catchment using historical change in impervious cover. Journal of Hydrology, 515, 59-70. doi: https://doi.org/10.1016/j. jhydrol.2014.04.011

Miranda, L. (2007). Evaluación de planes de desarrollo urbano, una alternativa de planeación en microcuenca Santa Rosa Jáuregui. Tesis de maestría. Universidad Autónoma de Querétaro. México.

Mobayed, K. (2009). Simulador de inundaciones en áreas urbanas. Un modelo de pronóstico con fines de alertamiento. Revista Digital CIENCIA@UAQ,2(1),72-90.

Morales, A. M. (2011). Propuesta de identificación de sitios para la infiltración de agua al acuífero de Querétaro. Tesis de Licenciatura. Universidad Nacional Autónoma de México. México.

Ochoa, M. (2019). Valoración de la calidad ambiental como estrategia para la planeación territorial, caso de estudio: Microcuenca el Pueblito-Joaquín Herrera, Querétaro. Tesis de maestría. Universidad Autónoma de Querétaro. México.

Opere, A. (2013). Floods in Kenya. In: P. Paron, D. Ochieng-Olago. \& C. Thine-Omuto. (Eds.), Developments in earth surface processes (pp. 315-330). Oxford: Elsevier.

Oviedo, B., Morán, E., Nájera, J., \& Bolívar, D. (2018). Implementación del sistema de alerta temprana SAT para evitar pérdidas humanas y materiales por inundaciones en las zonas periféricas de la ciudad de Quevedo. Universidad, Ciencia y Tecnología, 4, 80-91.

Peña, A. (2018). Estrategia de manejo para la mitigación del riesgo por inundaciones en la microcuenca San José El Alto, Querétaro. Tesis de maestría. Universidad Autónoma de Querétaro, Querétaro, México.

Programa de las Naciones Unidas para el Medio Ambiente (PNUMA). (2008). Perspectivas del medio ambiente urbano. GEO Zona Metropolitana de Querétaro. Querétaro: PNUMA. 
Sonia Oreano-Hernández - Juan Hernández-Guerrero Flooding within functional zones in the Santa Rosa Jauregui subwatershed Queretaro, Mexico

Reid, L. M. (1993). Research and cumulative watershed effects. USDA Forest Service General Technical Report PSW-GTR-141. Albany, California: Pacific Southwest Research Station, Forest Service, U.S. Department of Agriculture.

Rosgen, D. (1994). A classification of natural rivers. Catena, 22(3), 169199. doi: https://doi.org/10.1016/0341-8162(94)90001-9

Secretaría de Desarrollo Agrario, Territorial y Urbano (SEDATU) \& Consejo Nacional de Población (CONAPO). (2018). Sistema Urbano Nacional 2018. Ciudad de México: SEGOB/SEDATU/CONAPO.

Secretaría de Desarrollo Social (SEDESOL) \& Consejo Nacional de Población (CONAPO). (2012). Sistema Urbano Nacional 2012. Ciudad de México: SEGOB/SEDESOL/CONAPO.

Secretaría de Medio Ambiente y Recursos Naturales (SEMARNAT) \& Comisión Nacional del Agua (CONAGUA). (2015). Atlas del agua en México. Ciudad de México: SEMARNAT y CONAGUA.

Servicio Geológico Mexicano (SGM). (2018). Datos vectoriales de las cartas F14-10 y F14-C55. Recuperado de: https://www.sgm.gob. $\mathrm{mx} /$ GeoInfoMexGobMx/

Unidad Municipal de Protección Civil Querétaro (UMPCQ). (2015). Atlas de riesgos del municipio de Querétaro 2015. Querétaro: Municipio de Querétaro/UMPCQ/Sigema.

Unidad Municipal de Protección Civil Querétaro (UMPCQ). (2017). Programa estratégico para temporada de lluvias 2017. Querétaro: UMPCQ.

Universidad Autónoma de Querétaro (UAQ). (2009). Actualización del Plan Maestro Pluvial de la Zona Metropolitana de Querétaro (20082025). Querétaro, México: UAQ. Recuperado de: https://sistemamid. com/panel/uploads/biblioteca/2015-04-12_02-57-04119344.pdf

Valdés, A., \& Hernández, J. (2018). Zonas funcionales y unidades de paisaje físico-geográfico en la microcuenca Potrero de la Palmita, Nayarit, México. Revista Geográfica de América Central, 1(60), 189229. doi: https://doi.org/10.15359/rgac.60-1.7

Walker, L., MacDonald, R., McPherson, S., Barnes, C., Cipriano, C., Preston, G., Clarke, M., Chernos, M., Marcotte, D., Hopkins, C., \& Byrne, J. (2016). Elk River Flood Strategy. Alberta, Canada: Elk River Watershed Alliance. 
Weng, C., Ruhana, K., Zaini, M., Lee, L., \& Hin, C. (2016). Sustainable Urban Development. Malasia: Water Watch Penang \& Yokohama City University.

Xu, S., Nieto-Samaniego, A., Alaniz-Álvarez, S., \& Cerca-Martínez, L. (2011). Structural analysis of a relay ramp in the Querétaro graben, central Mexico: Implications for relay ramp development. Revista Mexicana de Ciencias Geológicas, 28(2), 275-289.

Zúñiga, E., \& Magaña, V. (2017). Vulnerability and risk to intense rainfall in Mexico: The effect of land use cover change. Investigaciones Geográficas, Instituto de Geografia, UNAM, (95), 1-18. doi: https:// doi.org/10.14350/rig.59465 
